3. The specific conductance of solutions of sodium and potassium at the same equivalent concentration is very nearly the same.

4. The equivalent conductance of saturated solutions or sodium and potassium in liquid ammonia is of the order of magnitude of that of such metals as strontium and iron.

5. The solubility of sodium and potassium in liquid ammonia at its boiling point has been found to be 5.367 and 4.866 mols of ammonia per atom for sodium and potassium respectively.

6. The results of this investigation furnish further evidence in support of the hypothesis that the conduction process in metals consists in a motion of negative carriers of sub-atomic dimensions, which carriers are the same for all metals.

WORCESTER, MASSAChUSETTS.

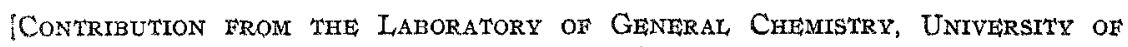
WISCONSTN.]

\title{
THE PREPARATION AND PROPERTIES OF THE PERSULFIDES OF HYDROGEN.
}

By JaMES H. WALTON AND LI,EWEIIXN B. PARSONS.

Received September 20, 1921.

Introduction.

When an excess of hydrochloric acid is added to a solution of a polysulfide of an alkali or one of the alkaline earths, a yellow oily liquid separates. Since the time of Scheele, who first prepared this substance, much work has been done on its preparation and the study of its properties. Among the more recent investigators, the work of Rebs, ${ }^{1}$ Bruni and Borgo, ${ }^{2}$ and Bloch and Höhn ${ }^{3}$ are worthy of note. The experiments of the latter authors are the most important in connection with this investigation. These investigators prepared the yellow oil by dissolving sulfur in a solution of sodium sulfide contained in a flask through which a stream of hydrogen was passed to prevent oxidation. The resulting solution was chilled and then run into a hydrochloric acid mixture consisting of one part of hydrochloric acid and one part of ice, surrounded by a mixture of ice and salt. Operating in this way they obtained a product with a density varying from 1.62 to 1.69 . The yellow oil when subjected to fractional distillation, yielded two products which on analysis gave the empirical formulas $\mathrm{H}_{2} \mathrm{~S}_{2}$ and $\mathrm{H}_{2} \mathrm{~S}_{8}$. Further experiments confirming the work of Bloch and Höhn were carried out by Schenck and Falcke. ${ }^{4}$

${ }^{1}$ Rebs, Ann. Chem., 246, 356 (1888).

a Bruni and. Borgo, Gazz. chim. ital., 38, 279, 292 (1907-8).

Bloch and Höhn, Ber., 41, 1961 (1908).

4 Schenck and Falcke, Ber., 41, 2600 (1908). 
The object of the present investigation was to improve the method for the preparation of the pure persulfides, and to study the chemical and physical properties of these compounds.

\section{Experimental.}

Preliminary.-To determine the effect of the common acids on a solution of sodium polysulfide, a solution of this substance similar to that used in the following experiments was prepared. This was cooled and slowly run into solutions of acetic, phosphoric, sulfuric, and hydrochloric acids respectively. Hydrochloric acid brought about the formation of the yellow oil; the other acids gave complete decomposition into sulfur and hydrogen sulfide.

The Preparation of the Crude Persulfide. - The methods given in the literature for the preparation of this substance are vague and difficult to repeat, many essential details being omitted. As a consequence, the modified method used by the authors is given in detail. Two $\mathrm{kg}$. of commercial c. P. sodium sulfide crystals was placed in a 3-liter round-bottomed flask, $300 \mathrm{~g}$. of flowers of sulfur added, and the two thoroughly mixed. Eight hundred cc. of water, enough to dissolve completely the resulting polysulfide at room temperature was then added. The flask was closed with a Bunsen valve, which obviates the necessity of passing hydrogen through the solution, and then heated on a water-bath for from 3 to 4 hours with occasional shaking. At the end of this period all the sulfur was dissolved. This polysulfide solution can be kept in a tightly stoppered vessel for several weeks without deteriorating sufficiently to influence the subsequent operations

Preliminary experiments showed that cold hydrochloric acid, sp. gr. 1.19, was satisfactory for decomposing the sodium polysulfide. The cooling and rate of bringing the two solutions together are of great importance. Fig. 1 illustrates the apparatus used for this purpose. $A$ and $A^{\prime}$ are galvanized iron containers insulated by excelsior. C is a specimen jar, diameter $25 \mathrm{~cm}$. This was surrounded by a mixture of ice and salt. Three liters of the concentrated acid was placed in the jar and agitated with a stirrer driven at the rate of about $100 \mathrm{r} . \mathrm{p.m}$. To aid in the cooling a few pieces of ice were always kept in the hydrochloric acid solution. During the reaction the temperature of the hydrochloric acid was kept between -4 and $-10^{\circ}$. The sodium polysulfide was added from $\mathrm{E}$ at a rate of about 3 liters per hour. Attention is called to the necessity of adding the polysulfide below the surface of the hydrochloric acid, this procedure minimizing the decomposition of the hydrogen persulfides. After about a liter and a half of the sodium polysulfide had been added, the persulfides began to rise in the form of a dark brown scum, and decompose with the evolution of hydrogen sulfide. At this point the addition of sodium 
polysulfide was stopped, the specimen jar was replaced with one containing a fresh portion of hydrochloric acid, and the operation continued until all the sodium polysulfide had been added. The persulfides, which were now largely present as an emulsion, settled on the bottom of the container in about 2 hours. This liquid was separated by means of a separatory funnel and washed several times with cold distilled water. In general $2 \mathrm{~kg}$. of sodium persulfide gave a yield of between 400 and $500 \mathrm{cc}$. of the crude product.

In drying the crude product obtained above, fused calcium chloride, previously treated with dry hydrogen chloride, had been used by previous investigators. Whenever the calcium chloride was used in this investigation, an appreciable amount of decomposition of the persulfides took place with the evolution of large amounts of hydrogen sulfide.

Phosphorus pentoxide has no action on the persulfides and so was used to dry them. Several $\mathrm{g}$. of this substance was allowed to stand in Fig. 1.-Apparatus for preparation of contact with the yellow liquid for a

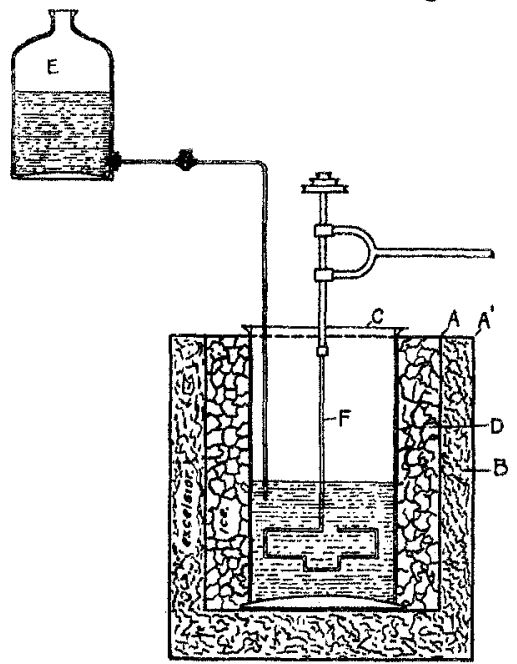
day or two with occasional shaking. At the end of that time the phosphorus pentoxide was removed by filtering through asbestos on a Büchner funnel. Fused powdered boric oxide may also be used to dry the yellow oil. This substance is inconvenient to prepare and offers no advantages over the phosphorus pentoxide. Analysis of the crude product prepared in this way showed that on decomposing it yielded between 19 and $21 \%$ of hydrogen sulfide. The compound $\mathrm{H}_{2} \mathrm{~S}_{5}$ evolves $20.98 \%$ of hydrogen sulfide. It is believed, however, that this crude product is a solution of sulfur in equilibrium with various sulfides of hydrogen and not a definite compound. This point will be the subject of further investigation.

Analysis of the Persulfides.-No rapid and accurate method for analyzing the persulfides has been described. Among those used may be mentioned (1) the decomposition of the persulfides by heat with subsequent determination of the hydrogen sulfide formed, (2) solution of the persulfide in an organic solvent such as toluene, addition of standard iodine and titration of excess of the iodine, (3) determination of the hydrogen by combustion and the sulfur by the Carius method. The published results of analyses by these methods are far from satisfactory. ${ }^{3}$ A long series of 
experiments carried ont to test Method 2 failed to give concordant results. In the analysis of the persulfides by the determination of the total hydrogen and the sulfur present, small experimental errors introduce very large errors in the empirical formulas computed. The method finally adopted consisted in dissolving a weighed amount of the hydrogen persulfide in carbon disulfide, adding acetone to decompose the persulfide, removing the acetone, carbon disulfide and hydrogen sulfide by evaporation, and weighing the sulfur. The hydrogen sulfide was then found by difference. The details of this method are as follows. Two to three $g$. of the sample was placed in a tared $75 \mathrm{~mm}$. quartz test-tube which was then corked and weighed at once. The tube was nearly filled with carbon disulfide, the contents poured into a tared $75 \mathrm{~mm}$. crystallizing dish and the tube washed out with the disulfide. The total volume of disulfide used was 20 to $30 \mathrm{cc}$. About $10 \mathrm{cc}$. of pure acetone was then added to the solution and the crystallizing dish covered with a watch glass. After the evolution of gas had ceased, the watch glass was rinsed with disulfide and the solution allowed to evaporate spontaneously, the process being complete in 3 to 4 hours. The residue of rhombic sulfur crystals was dried to constant weight at $90^{\circ}$.

A blank run made by evaporating $50 \mathrm{cc}$. of the mixed disulfide and acetone gave no weighable residue. The following table shows that the results obtained by this method are independent of the size of the sample and the volume of carbon disulfide used. Ten cc. of acetone was used in each case.

\begin{tabular}{|c|c|c|c|}
\hline & 1. & 2. & 3. \\
\hline $\mathrm{CS}_{2}, \mathrm{cc}, \ldots, \ldots \ldots \ldots, \ldots \ldots$ & 25 & 50 & 75 \\
\hline Sample, g.... & 2.2945 & 3.2243 & 2.1412 \\
\hline Sulfur residue, g.............. & 1.8476 & 2.5933 & 1.7240 \\
\hline $\mathrm{H}_{2} \mathrm{~S}$ evolved, g......... & 0.4469 & 0.6316 & 0.4172 \\
\hline $\mathrm{H}_{2} \mathrm{~S}$ in sample, \%. & 19.48 & 19.58 & 19.49 \\
\hline
\end{tabular}

This extremely accurate and rapid method was used in all the analyses made in this investigation.

Distillation of the Persulfide.-The distillation of the crude persulfide yields the compounds $\mathrm{H}_{2} \mathrm{~S}_{2}$ and $\mathrm{H}_{2} \mathrm{~S}_{3}$. The apparatus described by Bloch and Hohn may be used for this purpose; the yields obtained, however, were very unsatisfactory. Ordinary glass decomposes the persulfides of hydrogen, the action being due to the alkali in the glass. At temperatures necessary for distillation the decomposition is considerable. Several investigators have decreased this decomposition by treating the glass with hydrochloric acid. The advantage of quartz apparatus for distillation and also for preservation of the persulfides of hydrogen is shown by the following experiment. A quantity of the crude persulfide was placed in a small Pyrex flask that had been treated with hot conc. hydrochloric acid and dried by passing a stream of dry hydrogen chloride through it. An 
equal quantity of persulfide was placed in a clean dry quartz flask and the two allowed to stand under similar conditions for several days. In less than a week the persulfide in the Pyrex flask had decomposed to such an extent that crystals of sulfur were deposited. No sulfur crystals were deposited in the quartz flask after standing for a month.

The apparatus used in Fig. 2 is similar in principle to that of Bloch and Höhn. As far as possible quartz was used in its construction.

$B$ is a $250 \mathrm{cc}$. quartz distilling flask. The inner tube of the condenser, $E$, is of hard glass, $8 \mathrm{~mm}$. outside diameter. $\mathrm{C}$ is a $300 \mathrm{cc}$. quartz flask fitted with a 2-holed paraffined rubber stopper. By means of a hard glass tube it is connected with a $20 \mathrm{~cm}$. quartz test-ttbe which is also fitted with a paraffined rubber stopper. A is a $500 \mathrm{cc}$. hard glass distilling flask.

In carrying out the distillation the flask B was heated to $120^{\circ}$ in a glycerine bath and the system exhausted by means of a vacuum pump to a pressure of 3 to $5 \mathrm{~mm}$. $C$ was cooled by the water from the condenserwhichran out through a $Y$-tube. $D$ was cooled in the first experiments by solid carbon dioxide and ether as described by Bloch and Höhn. Salt and ice mixture was found to be quite satisfactory, however, and this mixture was used in

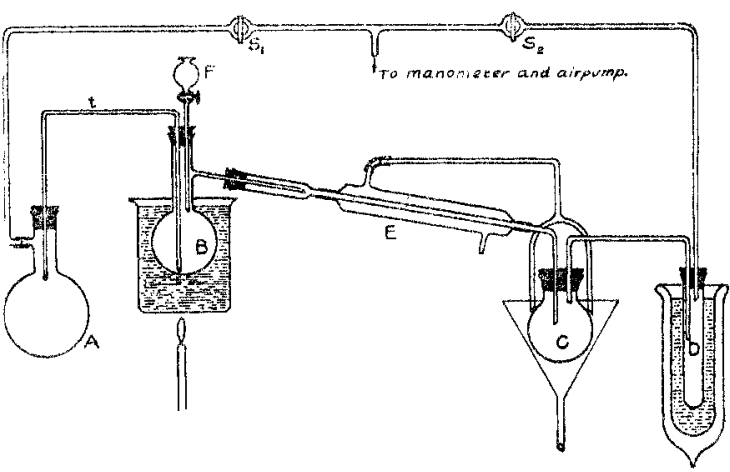

Fig. 2,-Distilling apparatus for persulfides.

the later experiments. Ten to $15 \mathrm{cc}$. of the crude persulfide was rutn into $\mathrm{B}$ through the separatory funnel, $\mathrm{F}$, whereupon the persulfides began to distil slowly. After 10 minutes the residue in B consisted mainly of sulfur dissolved in persulfide. This was removed by closing the cock $S_{1}$ and opening $S_{2}$, whereupon the residue was drawn over into $A$. Inasmuch as this residue solidifies on cooling, the tube $t$ had to be carefully heated during this operation. After the residue had been removed from the flask, $S_{1}$ was closed, $S_{2}$ opened, more persulfide added, and the distillation carried on as before.

The yield by distilling the crude product depends upon many factors, the most important of which is the age of the sample. Two volumes of the freshly prepared persulfides usually gave 1 volume of distillate; $2 / 3$ of this was hydrogen trisulfide and $1 / 3$ hydrogen disulfide.

In the later experiments it was found that a vacuum of 20 to $25 \mathrm{~mm}$. could be used in the above distillation; as a consequence a water suction pump can be used with entire satisfaction. 
Hydrogen Trisulfide. - This substance collects in Receiver $C$ in a high state of purity, as is shown by the following results of analysis.

\begin{tabular}{|c|c|c|c|}
\hline Sam & $\begin{array}{c}1 . \\
28576\end{array}$ & $\begin{array}{c}2 . \\
17875\end{array}$ & $\begin{array}{c}3 . \\
3.7798\end{array}$ \\
\hline Sulfur residue, $g . \ldots \ldots \ldots$ & 1.8727 & 1.1740 & 2.4838 \\
\hline $\mathrm{H}_{2} \mathrm{~S}$ evolved, $\mathrm{g} . .$. & 0.9848 & 0.6135 & 1.2960 \\
\hline $\mathrm{H}_{2} \mathrm{~S}$ in sample, $\%$ & 34.46 & 34.42 & 34.28 \\
\hline Calculated $\mathrm{H}_{2} \mathrm{~S}$ in $\mathrm{H}_{2} \mathrm{~S}_{3}, \% \ldots \ldots$ & 34.69 & $\ldots$ & . \\
\hline
\end{tabular}

Hydrogen trisulfide is a mobile, yellow, oily, liquid with an odor similar to that of camphor and sulfur monochloride. Its vapors attack the eyes and nose strongly. On cooling the trisulfide, the yellow color dis. appeared, this behavior being exactly analogous to that of yellow rhombic sulfur which becomes pure white at the temperature attainable with solid carbon dioxide snow and ether. The trisulfide was soluble in benzene, toluene, chloroform, carbon disulfide, ether, and heptane, while alcohols, ketones, nitrobenzene, aniline and pyridine caused it to decompose catalytically. In any of the above mentioned solvents it was noted that small quantities of impurities caused the trisulfide to decompose with the deposition of white gleaming crystals of nacreous sulfur which, on standing changed rapidly into ordinary rhombic sulfur.

To determine the melting point of the trisulfide, some of it was cooled in a bath of carbon dioxide snow and ether to a temperature of $-78^{\circ}$ as registered on a toluene thermometer. On cooling, the trisulfide behaved like a glass, becoming more and more viscous, until solidification took place. No definite melting point was noted. The quartz tube containing the trisulfide was removed from the freezing mixture and allowed to warm up slowly. The thermometer remained constant for about a minute at $-52^{\circ}$ to $-53^{\circ}$. This is the melting point of the trisulfide as given by Bloch and Höhn.

The hydrogen trisulfide distilled readily in the vacuum apparatus, with little or no foaming. In one case $100 \mathrm{cc}$. of the trisulfide was distilled and not more than $10 \mathrm{cc}$. of the sulfur and persulfide residue was left behind in the distilling flask. About $15 \mathrm{cc}$. of the disulfide was obtained at the same time.

Attempts were made to distil the trisulfide under atmospheric pressure. A small quartz still with ground joints was available; this was fitted with a small quartz condenser. Ten cc. of the compound was placed in the distilling flask and carefully heated. The whole mass foamed, evolving hydrogen sulfide, and leaving behind a thick gummy solution of sulfur in persulfide. One or two drops of distillate were obtained, but it was never possible to obtain a sample of sufficient size for analysis.

Hydrogen Disulfide.-In the second container, cooled with a mixture of carbon dioxide snow and ether, a compound of the approximate composition $\mathrm{H}_{2} \mathrm{~S}_{2}$ collected. The compound obtained never showed by analysis 
the composition $\mathrm{H}_{2} \mathrm{~S}_{2}$, being always 1 or $2 \%$ low in hydrogen sulfide content. The following is a typical analysis.

$\begin{array}{lcc} & & \\ \text { Sample, } \mathrm{g} \ldots \ldots \ldots \ldots \ldots \ldots \ldots \ldots & 3.4490 & 3.2283 \\ \text { Sulfut residue, g. } \ldots \ldots \ldots \ldots \ldots \ldots & 1.7340 & 1.6220 \\ \mathrm{H}_{2} \mathrm{~S}, \mathrm{~g} \ldots \ldots \ldots \ldots \ldots \ldots \ldots \ldots & 1.7150 & 1.6063 \\ \% \mathrm{H}_{2} \mathrm{~S} \ldots \ldots \ldots \ldots \ldots \ldots \ldots \ldots & 49.72 & 49.76 \\ \mathrm{H}_{2} \mathrm{~S} \text { calc. in } \mathrm{H}_{2} \mathrm{~S}_{2} \% \ldots \ldots \ldots \ldots \ldots & \ldots \ldots & 51.20\end{array}$

These results indicated that the disulfide contained some dissolved trisulfide. This led to an attempt to distil the sample, obtained in the vacuum distillation, at ordinary pressures. The small quartz still, previously employed for the attempted distillation of the trisulfide, was used. Some of the disulfide was placed in the still, which was heated carefully with a micro burner. The disulfide distilled without foaming or bubbling, and was readily condensed in the small condenser which was kept at a temperature of about $15^{\circ}$ by running water. The disulfide did not all distil, about $1 / 5$ of the original volume remained behind as a gummy viscous mass. Samples of the distillate were collected directly in the quartz weighing tube and analyzed immediately. The following results were obtained.

\begin{tabular}{|c|c|c|}
\hline Sample, g. . & 1.9172 & 2.0351 \\
\hline Sulfur residue, g..... & 0.9404 & 0.9981 \\
\hline Hydrogen sulfide, g. & 0.9768 & 1. 0370 \\
\hline Sulfur in sample, $\%$. & 50.94 & 50.95 \\
\hline Hydrogen stlfide, in $\mathrm{H}_{2} \mathrm{~S}_{2}, \%$... & & 51.20 \\
\hline
\end{tabular}

The hydrogen disulfide was a practically colorless mobile oil with a much more severe and irritating odor than the trisulfide. When the disulfide was being distilled under atmospheric pressure the vapors were extremely irritating, causing tears and a smarting sensation in the nostrils. The disulfide dissolved in the same solvents as the trisulfide; it was, however, much more sensitive to small amounts of impurity than the latter. This was especially noticeable when acetone was added to the carbon disulfide solution in the analytical procedure. It was consequently necessary to be very cautious in adding the acetone in order not to have the hydrogen sulfide evolved so rapidly that the liquid would bubble over.

The boiling point of the disulfide was determined by placing some of the distilled product in a small quartz tube equipped with a two-holed rubber stopper carrying a sensitive thermometer. On gently heating, the disulfide boiled at $74.5^{\circ}$, checking the value obtained by Bloch and Höhn.

The melting point of the disulfide was determined by a method similar to the one used for the trisulfide; the cooling agent used was liquid air. The slowing up in the rise of the toluene thermometer used came at $-88^{\circ}$ to $-90^{\circ}$ in several trials. The disulfide behaved like the trisulfide on cooling, showing no sharp solidification point. 
Action of Trisulfide Solutions on Inorganic Salt Solutions.-Because of the extreme ease of decomposition of the disulfide, the trisulfide was used in the experimental work which follows. The action of an ether solution of the trisulfide on solutions of various inorganic salts dissolved in the same solvent was tested. As a check on the experiments, similar solutions of the salts were treated with a saturated ether solution of hydrogen sulfide. Copper oleate gave a red-brown colloidal precipitate; ferric chloride solution gave a white precipitate, which redissolved; stannic iodide gave a buff precipitate; silver nitrate gave no change; mercuric bromide gave a yellow precipitate. In every case the saturated ether solution of hydrogen sulfide gave a similar result. It was concluded that the behavior of the trisulfide in ether solution is essentially the same as that of hydrogen sulfide.

Action of the Trisulfide on Metallic Oxides and Oxidizing Agents.The effect of adding small amounts of the trisulfide to a number of metallic oxides was observed by pouring some of the trisulfide upon a small amount of the oxide placed on a carefully cleaned watch glass.

Silver oxide, copper oxide, lead peroxide and mercuric oxide caused violent decomposition of the trisulfide; the heat evolved was sufficient to ignite the persulfide.

Iead oxide, stannic oxide and magnetite brought about a violent decomposition of the trisulfide.

Arsenious oxide, arsenic oxide, ferric oxide, zinc oxide, barium peroxide and manganese dioxide caused only a slow decomposition.

The oxidizing agents potassium permanganate and potassium dichromate were found to decompose the trisulfide rapidly. The permanganate generated sufficient heat to ignite the persulfide.

Action of the Trisulfide on Inorganic Salts.-A number of salts were treated with small amounts of the trisulfide. The sulfates of ferrous iron, aluminum, nickel, zinc and manganese gave a very slow decomposition of the trisulfide. Anhydrous copper sulfate, however, decomposed the trisulfide rapidly, the whole mixture turning dark.

The nitrate of aluminum gave only a slow decomposition while the nitrates of lead, silver and copper caused a rapid decomposition of the persulfide. Ferric nitrate behaved in a peculiar manner; for a time no visible decomposition took place and then suddenly an increasingly violent reaction began, as if it were an autocatalytic effect.

The chlorides of manganese, sodium, cadmium, ammonium and lead decomposed the trisulfide only slowly. The chloride of antimony dissolved with the consequent decomposition of the trisulfide. The chlorides of copper and lead turned dark with accompanying decomposition of the trisulfide.

The acetate, bromide and oleate of copper decomposed the trisulfide 
rapidly turning dark at the same time. Antimony tri-iodide dissolved in the persulfide. The mixture turned red and decomposed.

Massive metals did not decompose the persulfide rapidly. They became coated with the sulfide of the metal and the reaction ceased. Powdered arsenic, antimony and zinc and iron, however, brought about rapid decomposition.

It was found that the persulfide was not decomposed by finely ground quartz which had been washed with hydrochloric acid and carefully dried. Neither did boric oxide nor phosphorus pentoxide bring about decomposition. It is to be noted that all copper salts decompose the persulfide as do most lead salts.

In order to see whether it would not be possible to dissolve some of the sulfides of copper and arsenic in the pure hydrogen trisulfide and thus obtain the free sulfo acids of these metals, a small amount of freshly precipitated, well washed and dried copper sulfide was added to the trisulfide contained in a quartz tube. The sulfide did not dissolve in the persulfide and was filtered off. A similar result was obtained with fresh arsenic trisulfide.

The Decomposition of Hydrogen Trisulfide in a Sealed Tube.-To observe the behavior of the trisulfide under pressure, some of it was sealed in a thick glass tube which had been scrupulously cleaned and carefully dried with hydrogen chloride. The hydrogen sulfide content of the sample was $34.4 \%$. The sealed tube was allowed to stand in a hood under ordinary laboratory conditions. At the end of 3 days small crystals of sulfur began to appear, while at the end of 5 days there were two liquid layers noticeable, the one heavy and viscous, the other light, mobile and colorless, presumably hydrogen sulfide. There were large crystals of sulfur in the tube. After 2 days more, the viscous layer had completely disappeared and the tube contained only several large rhombic crystals of sulfur and liquid hydrogen sulfide, with possibly dissolved persulfide.

Solubility of Hydrogen Trisulfide in Hydrogen Sulfide.-Bruni and Borgo state that hydrogen sulfide is insoluble in the persulfides. They claimed to have liquefied hydrogen sulfide and found that the persulfide did not dissolve therein. In order to establish this fact definitely, hydrogen trisulfide $\left(\mathrm{H}_{2} \mathrm{~S}\right.$ content $34.4 \%$ ) was carefully introduced into a clean, heavy-walled tube. The tube was placed in a freezing mixture of carbon dioxide snow and ether. By passing a current of hydrogen sulfide into the cooled tube, the gas could be easily liquefied. About two volumes of the liquid hydrogen stifide was collected above the trisulfide and the tube was sealed off. As soon as the trisulfide melted it completely dissolved in the hydrogen sulfide. On warming to room temperature, the solution was a pale straw color. When the tube stood for 5 days large crystals of rhombic sulfur separated, but there was no separation at any time of the persulfide such as was noted in the previous case when hydrogen 
trisulfide alone was sealed off. Bruni and Borgo never obtained hydrogen trisulfide, so possibly, working with persulfides containing much dissolved sulfur, the liquid hydrogen sulfide might not dissolve the compounds completely. The previously noted fact that the trisulfide alone gave a layer of liquid hydrogen sulfide and also a layer of the persulfide containing dissolved sulfur, would point to this explanation as being the correct one for Bruni and Borgo's results.

Solubility of Sulfur in Hydrogen Trisulfide.-To determine the amount of sulfur which would dissolve in the trisulfide, several samples of trisulfide were saturated with sulfur and the hydrogen sulfide content of the solution obtained. Sulfur dissolved until the hydrogen sulfide content of the system was between 8 and $9 \%$. The temperature of the samples varied between $17^{\circ}$ and $20^{\circ}$. Calculated on the basis that all the hydrogen sulfide was present as the trisulfide one mole of the trisulfide dissolved 8 moles of sulfur.

\section{Summary.}

1. The conditions under which pure hydrogen disulfide and hydrogen trisulfide may be prepared in quantity have been determined.

2. A new method for analyzing the persulfides of hydrogen has been devised. This method is rapid and accurate.

3. The hydrogen persulfides decompose most slowly when stored in quartz vessels.

4. The persulfides of hydrogen are decomposed catalytically by many strbstances, both inorganic and organic. Of the latter, ketones, aldehydes, alcohols, amino substitution products and nitro derivatives are specially effective.

5. It has been shown that hydrogen trisulfide and liquid hydrogen sulfide are miscible, thus disproving the statement of Bruni and Borgo to the contrary.

6. Hydrogen disulfide freezes at a temperature between $-88^{\circ}$ and $-90^{\circ}$. Madrson, Wrsconsin. 